\title{
Rab25 acts as an oncogene and participates in the regulation of aerobic glycolysis via PKM2 in gastric adenocarcinoma
}

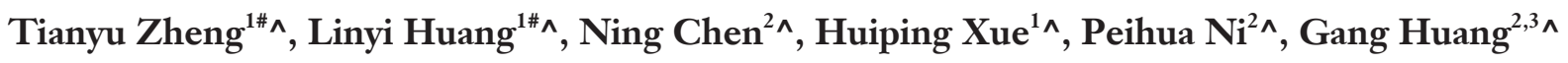 \\ ${ }^{1}$ Shanghai Institute of Digestive Disease, Renji Hospital, Shanghai Jiao Tong University School of Medicine, Shanghai, China; ${ }^{2}$ Department of \\ Laboratory Medicine, Ruijin Hospital, Shanghai Jiao Tong University School of Medicine, Shanghai, China; ${ }^{3}$ Shanghai Key Laboratory of Molecular \\ Imaging, Shanghai University of Medicine and Health Sciences, Shanghai, China \\ Contributions: (I) Conception and design: H Xue, P Ni, G Huang; (II) Administrative support: H Xue, P Ni, G Huang; (III) Provision of study \\ materials or patients: H Xue, P Ni; (IV) Collection and assembly of data: T Zheng, N Chen, L Huang; (V) Data analysis and interpretation: T \\ Zheng, N Chen, L Huang; (VI) Manuscript writing: All authors; (VII) Final approval of manuscript: All authors. \\ "Those authors contributed equally to this work. \\ Correspondence to: Prof. Huiping Xue, MD, PhD. Shanghai Institute of Digestive Disease, Renji Hospital, Shanghai Jiao Tong University School of \\ Medicine, Shanghai, China. Email: huiping_xue@163.com; Prof. Peihua Ni, PhD. Department of Laboratory Medicine, Ruijin Hospital, Shanghai \\ Jiao Tong University School of Medicine, Shanghai, China. Email: nipeihua@126.com; Prof. Gang Huang, MD, PhD. Shanghai Key Laboratory of \\ Molecular Imaging, Shanghai University of Medicine and Health Sciences, Shanghai, China. Email: huangg@sumhs.edu.cn.
}

Background: Rab25, a hub node of protein-protein interaction networks, has become one of the most popular tumor-associated proteins. Pyruvate kinase $(\mathrm{PK})$ is the main rate-limiting enzyme in the glycolysis pathway and plays a significant role in the regulation of Warburg effect. In this study, we aimed to characterize the expression and function of Rab25 in gastric adenocarcinoma (GAC) and verify our hypothesis experimentally that Rab25 may participate in the regulation of aerobic glycolysis via PKM2 (a subtype of PK) in GAC.

Methods: The impact of Rab25 expression on patient overall survival was estimated using the KaplanMeier method. Rab25 expression was silenced or increased respectively by lentivirus-mediated transfection. To assess the role of Rab25 in cell viability in vitro, cell proliferation and migration experiments were performed. Levels of pyruvate and lactic acid were detected by kits. Immunofluorescence analysis and CoImmunoprecipitation were performed to explore the interaction between Rab25 and PKM2 protein.

Results: High Rab25 expression was associated with reduced patient overall survival. Silencing Rab25 inhibits GAC cells proliferation and overexpressing Rab25 promotes cell proliferation and migration in gastric cells in vitro. The study revealed that Rab25 participates in the positive regulation of aerobic glycolysis in GAC cells. Rab25 protein and PKM2 protein co-located on the cell membrane and could bind to each other in GAC cells. Rab25 is a positive regulator of PKM2 and Rab25 up-regulation promotes phosphorylation of PKM2.

Conclusions: In human GAC, Rab25 predicts poor prognosis and regulates aerobic glycolysis via phosphorylating PKM2-Y105.

Keywords: Rab25; gastric adenocarcinoma (GAC); PKM2; aerobic glycolysis; phosphorylation

Submitted Jul 25, 2020. Accepted for publication Dec 17, 2020.

doi: $10.21037 /$ tcr-20-2597

View this article at: http://dx.doi.org/10.21037/tcr-20-2597

\footnotetext{
^ ORCID: Tianyu Zheng, 0000-0001-9976-127X; Linyi Huang, 0000-0002-8575-8587; Ning Chen, 0000-0001-6487-539X; Huiping Xue, 0000-0001-9470-1526; Peihua Ni, 0000-0002-1647-1996; Gang Huang, 0000-0003-1939-3978.
} 


\section{Introduction}

Gastric cancer is a common malignant tumor, with over 1 million new cases and more than 780 thousand deaths worldwide annually, making it the fifth most frequently diagnosed cancer and the third leading cause of cancer death (1). Gastric adenocarcinoma (GAC) accounts for about $95 \%$ of all gastric malignant tumors. Despite the continuous improvement of digestive endoscopy, surgical techniques and clinical treatment of GAC, the prognosis of patients with GAC is still not optimistic (2). The diagnostic rate of early stage of GAC is low and its prognosis has not been fundamentally improved. Therefore, it is particularly important to explore the molecular mechanism of the carcinogenesis and progression of GAC and formulate new strategies for its prevention and treatment.

Multiple factors and complicated procedures participate in stomach tumorigenesis. It involves not only the abnormal regulation of signaling transduction pathways and gene expressions, but also the metabolic changes in tumor cells (3). Because cellular sugar metabolism is the most basic form of metabolism, the abnormality of glucose metabolism in tumor cells, unraveled in the Warburg effect, is considered to be the pivotal marker of tumor (4). In aerobic conditions, the normal cells mainly supply energy through the oxidative phosphorylation of glucose. In hypoxia, normal cells provide energy with glycolysis, where glucose is converted to pyruvate, the latter of which is converted to lactic acid. Unlike normal cells, tumor cells, even under aerobic conditions, produce large amounts of lactic acid via aerobic glycolysis instead of mitochondrial oxidative phosphorylation, the former known as the Warburg effect (5). The Warburg effect represents a shift of using glucose from oxidative phosphorylation to glycolysis, universally acknowledged to be the most important feature of cancer. Thus, it is of great practical significance to elucidate the molecular mechanism of gastric carcinogenesis through probing the metabolic abnormalities implicated in GAC cells so as to lay down more effective modalities for the prevention and treatment of GAC.

The Rab protein family, the largest subfamily of the Ras superfamily, is composed of small molecule GTP binding proteins. They participate in many key processes during cellular endocytosis and membrane recycling, and predominantly regulate the key process of vesicle transports and protein exchanges in cells (6). Rab25, a newly discovered Rab family protein, is located in lq22 and belongs to a member of the Rab11 subfamily (7). Rab25, a hub node of protein-protein interaction networks, has become one of the most popular tumor-associated proteins (8). Mounting evidence suggests that Rab25 particularly plays a vital role in tumorigenesis (9-12). It has been consecutively reported that Rab25 mRNA or protein was found to be highly expressed in most epithelial tumors including ovarian $(13,14)$, luminal B breast (15), testicular (16), bladder (17), prostatic (18) and liver tumors (19). Predominantly reported, Rab25 acts as an oncogene, especially in ovarian cancer, the level of whose expression in tumor tissue samples of patients is negatively correlated with their survival rate (20). As an oncogene, Rab25 has been shown to increase the proliferation, invasion, and metastasis of ovarian cancer cells in vitro. It also has been demonstrated that Rab25 increases tumorigenicity and decreases tumor cell apoptosis in vivo animal models (21). Recently, one interesting study found that Rab25 can promote the proliferation and invasion of ovarian cancer cells via the regulation of their energy metabolism (22). The study insinuates the possibility that Rab25 may regulate glycometabolism in ovarian cancer cells, which may constitute a novel mechanism of Rab25 in carcinogenesis. Given that the major form of glucose metabolism is aerobic glycolysis in tumors, we focused on the pathway of aerobic glycolysis.

Pyruvate kinase $(\mathrm{PK})$ is the main rate-limiting enzyme in the glycolysis pathway and plays a significant role in the regulation of glycolysis and Warburg effect (23). Mammalian PKs are encoded by two genes (PKLR and PKM), and each can generate two isoforms, respectively (PKL and PKR; PKM1 and PKM2) (24). In the process of malignant transformation of adult cells, the expression of PKM2 was reported to be particularly increased and play an important role in cell metabolic reprogramming (25). In addition, PKM2 was also reported to promote tumor growth by inhibiting tumor cell apoptosis or transforming tumor metabolism into aerobic glycolysis and bringing nutrients to biosynthesis (26-28). In view of the vital function of PKM2 in tumor metabolism, it is worth exploring that PKM2 acts as a target of tumor regulation. There is growing evidence that oncogenes and tumor-related signaling pathways are more specific to the regulation of glucose metabolismrelated enzymes. Therefore, it is very important to explore the effects of oncogene on the activity of metabolic enzyme in order to find a more specific target of chemotherapeutic drugs (29).

In this study, we explored the expression and function of Rab25 in GAC. In view of the fact that Rab25 is a gene 
Table 1 Sequence of primers used in this study

\begin{tabular}{ll}
\hline Gene & \multicolumn{1}{c}{ Primer sequence $\left(5^{\prime} \rightarrow 3^{\prime}\right)$} \\
\hline Rab25 $(F)$ & GCCCTGGACTCTACCAATGTTA \\
Rab25 $(R)$ & GCTGTTCTGTCTCTGCTTGGACAC \\
PKM2 $(F)$ & CGCTGGATAACGCCTACA \\
PKM2 $(R)$ & CATCAAACCTCCGAACCC \\
$\beta$-actin $(F)$ & ACCAACTGGGACGACATGGAGAA \\
$\beta$-actin $(R)$ & GGGATAGCACAGCCTGGATAGCA
\end{tabular}

$\mathrm{F}$, forward; $\mathrm{R}$, reverse.

that has a significant effect on the energy metabolism of cancer, we explore whether it affects tumor metabolism by regulating PKM2 and further affects the development of GAC. Through the exploration of Rab25, Warburg effect and PKM2, we aimed to validate our hypothesis that Rab25 participates in glycolysis pathway via PKM2.

We present the following article in accordance with the MDAR checklist (available at http://dx.doi.org/10.21037/ tcr-20-2597).

\section{Methods}

\section{Cell lines and cell culture}

Human GAC cell lines (AGS, SGC-7901, MGC-803, and NCI-N87) and normal human gastric mucosal epithelial cell line GES-1 were purchased from the Type Culture Collection of the Chinese Academy of Sciences (Shanghai, China). Cells were cultured in RPMI 1640 medium with $10 \%$ fetal bovine serum, $100 \mathrm{U} / \mathrm{ml}$ penicillin sodium, and $100 \mathrm{mg} / \mathrm{ml}$ streptomycin sulfate in humidified $5 \% \mathrm{CO}_{2}$ at $37^{\circ} \mathrm{C}$.

\section{RNA interference and stable cell line generation}

To construct the stable Rab25-overexpressing and Rab25silencing cells, the recombinant lentivirus pHBLV- Rab25 encoding Rab25 or pHBLV-n.c (negative control) and recombinant lentivirus pHBLV-shRab25 or pHBLV-shn. c were purchased from Hanbio (Shanghai, China). The shRNAs were cloned into $\mathrm{pHBLV-U6-Puro} \mathrm{lentiviral} \mathrm{vector}$ (Hanbio; China). The target sequences were shRab25-1, 5'-GATGGCTGAAGGAGCTCTATG-3'; shRab25-2, 5'- GCCTTTGAGACTGTCCTGAAA-3'. Lentivirus infection was conducted according to the manufacturer's protocols. In brief, the differentiated GES-1 and AGS cells were seeded into a 6-well plate and cultured overnight to reach $70 \%$ confluence. Subsequently, cells were transfected with above different lentiviruses in the presence of ploybrene ( $8 \mu \mathrm{g} / \mathrm{mL}$; Sigma-Aldrich; USA) for $24 \mathrm{~h}$. After $24 \mathrm{~h}$, each culture medium was removed and each fresh medium was added. At $72 \mathrm{~h}$ after transduction, puromycin $(5 \mathrm{mg} / \mathrm{mL}$ ) was added to each medium for stable cell line selection. The empty lentivector lenti-puromycin was used as negative control. After antibiotic selection for 3 weeks, stable cells were obtained. In addition, we performed the transfection of PKM2 siRNA (siPKM2) and Control siRNA (siCtrl) plasmid using the Lipofectamine 2000 reagent (Invitrogen) according to the manufacturer's instructions.

\section{$R N A$ isolation and quantitative real-time PCR}

Total RNAs were extracted from cell lines with Trizol reagent (Invitrogen) according to the manufacture's protocol. To obtain cDNA, PrimeScript ${ }^{\mathrm{TM}} \mathrm{RT}$ reagent kit (TaKaRa) was used for reverse transcription. Expression levels of candidate genes and $\beta$-actin were determined by quantitative real-time PCR using a Qiagen Rotor-Gene Q system (Qiagen, Inc.) and the levels of gene expression were calculated using the $2^{-\Delta \Delta \mathrm{Ct}}$ method. All reactions were run in triplicate. Sequences of primers are listed in Table 1.

\section{Protein extraction and western blot analysis}

Cells were washed twice with ice-cold PBS and lysed in RIPA buffer for $15 \mathrm{~min}$. Cell debris was removed by centrifugation at $12,000 \mathrm{~g}$ for $30 \mathrm{~min}$ at $4{ }^{\circ} \mathrm{C}$. Total protein lysates were quantitated using $\mathrm{BCA}$ protein assay kit (Pierce Biotechnology Inc). Equal amount of total protein lysates was separated with $12 \%$ sodium dodecyl sulfatepolyacrylamide gels and transferred to a polyvinylidene difluoride membrane. The membrane was blocked with $5 \%$ BSA, followed by incubation overnight at $4{ }^{\circ} \mathrm{C}$. The following antibodies were used for immunoblotting: $\beta$-Actin (1:1,000; Cell Signaling Technology; 3700), Rab25 (1:1,000; Cell Signaling Technology; 13048), PKM2 (1:2,000; R\&D SYSTEMS; MAB7244), PKM2-p-Tyr105 (1:2,000; Signalway Antibody; 12821), and PKM2-p-Tyr (1:1,000; PTM Biolabs; PTM-701). Goat anti-mouse or goat anti-rabbit IgG horseradish peroxidase (Cell Signaling Technology) was used as secondary antibodies. Finally, the pictures were visualized using SuperSignal ${ }^{\mathrm{TM}}$ West Pico 
Chemiluminescent Substrate (Thermo Scientific).

\section{Cell proliferation assay}

Cell proliferation was determined by survival cell count assay. Cells were seeded in 6-well plate at approximately 2000 cells per well and incubated for 5 days at $37^{\circ} \mathrm{C}$ with $5 \%$ $\mathrm{CO}_{2}$ in a humidified incubator. Survival cells were quantified by cell counter (Beckman Coulter, Inc.) every $24 \mathrm{~h}$.

\section{Colony-formation assay}

AGS cells $\left(5 \times 10^{2}\right)$ stably expressing shRNA targets against Rab25 and their relative control cells were seeded in six-well plates with each $2 \mathrm{~mL}$ culture medium. GES-1 pHBLV-Rab25 stable cells and their relative control cells were seeded in six-well plates with 500 cells/well under the same culture conditions. The cells were cultivated for 2-3 weeks until their colonies were visible. Cells were washed twice with PBS and fixed with pure methanol at room temperature for $15 \mathrm{~min}$. Then the cells were stained with coomassie brilliant blue dye and incubated at room temperature for $30 \mathrm{~min}$. Coomassie brilliant blue dye was carefully removed and the plates in $\mathrm{ddH}_{2} \mathrm{O}$ were immersed to rinse off coomassie brilliant blue dye. After the plates were dried at room temperature, the number of colonies was counted.

\section{Wound healing assay}

Cell migration was evaluated using wound healing assay. Briefly, $5 \times 10^{5}$ cells/well were plated in a 6-well plate and cultured overnight to yield a confluent monolayer. The cells were then wounded with a $200 \mu \mathrm{L}$ pipette tip. The remaining cells were washed three times with PBS and then cultured with RPMI 1640 medium without FBS. Photographs were taken at 0,6 , and 12 hours.

\section{Soft-agar colony formation assay}

First of all, $2 \mathrm{~mL}$ of $1.2 \%$ agar containing $2 \times$ culture medium was added to each well of a 6 -well plate. After the bottom agar was solidified, $2 \mathrm{~mL}$ of a mixture of $0.7 \%$ agar and 500 cells in $2 \times$ culture medium around $40{ }^{\circ} \mathrm{C}$ was added on top of the $1.2 \%$ agar. One hundred microliters of medium were added twice weekly to prevent desiccation of the upper layer of agar. After 2 weeks, colonies were counted under the electron microscope.

\section{Cellular metabolic analysis.}

The stable cell lines with Rab25 overexpression or knockdown were cultured at $37^{\circ} \mathrm{C}$ with $5 \% \mathrm{CO}_{2}$ in a humidified incubator, and intracellular lactate levels were measured using a Lactate Assay Kit (Nanjing Jiancheng Bioengineering Institute; A019-2). Cellular pyruvic acid levels were measured using a Pyruvate Assay Kit (Nanjing Jiancheng Bioengineering Institute; A081). Cellular PK activity was measured using the PK enzyme activity dipstick assay kit (Nanjing Jiancheng Bioengineering Institute; A076-1).

\section{IHC staining and score system}

Human GAC tissue specimens and corresponding nonmalignant gastric tissue specimens along with their clinical and pathological data were collected and confirmed in Renji hospital, Shanghai Jiao Tong University School of Medicine. Stomach specimens were fixed in $4 \%$ paraformaldehyde and embedded in paraffin. Tissue sections were stained with haematoxylin and eosin in accordance with standard methods. For IHC, tissue sections $(4 \mathrm{~mm})$ were subjected to heat-mediated antigen retrieval (citrate buffer, $\mathrm{pH} 6$ ). Primary antibodies were applied and incubated at $4{ }^{\circ} \mathrm{C}$ overnight. The following primary antibodies were used: Rab25 (1:100; proteintech; 13189) and PKM2 (1:800; proteintech; 60268). The immunoreactive score (IRS) was used to quantify the IHC staining, giving a range of 0 to 12 as a result of multiplication of positive cell proportion scores $(0-4)$ and staining intensity scores $(0-3)$ as previously reported (30). IHC staining and statistical analyses were performed in a double-blind manner.

\section{Immunofluorescence analysis}

AGS cells were cultured on glass coverslips in a 24-well plate, fixed with $4 \%$ paraformaldehyde, permeabilized using $0.1 \%$ Triton $\mathrm{X}-100$, washed with $1 \times$ PBS, and blocked with $5 \%$ bovine serum albumin. Then the samples were incubated with Rab25 antibody (1:200; Cell Signaling Technology;13048) and/or PKM2 antibody (1:200; R\&D SYSTEMS; MAB7244) at $4{ }^{\circ} \mathrm{C}$ overnight, followed by staining with Alexa Fluor 568 Goat anti-Rabbit IgG (1:200; Thermo Fisher Scientific; 1738303) and Alexa Fluor 647 Goat anti-Mouse IgG (1:200; Thermo Fisher Scientific; 1764240). Coverslips were mounted with vectashield mounting medium (Vector Laboratories, CA 94010) 
containing diamidino-2-phenylindole (DAPI) nuclear stain and examined under a confocal laser scanning microscope.

\section{Co-immunoprecipitation}

The AGS cells were trypsinized, washed with ice-cold PBS, lysed with IP lysis buffer (Beyotime Biotechnology; P0013) plus PMSF and cleared by centrifugation. To immunoprecipitate endogenous proteins, lysate $(500 \mu \mathrm{g})$ was mixed with $2 \mu \mathrm{g}$ PKM2 (proteintech; 60268) and its relative $\operatorname{IgG}$ (Beyotime Biotechnology; A7082) antibodies overnight at $4{ }^{\circ} \mathrm{C}$. The next day, UltraLink Immobilized Protein A beads (Beyotime Biotechnology; P2015) were added to the antigen-antibody complex and incubated for two hours at room temperature with rotation. Beads were recovered by low speed centrifugation and washed with immunoprecipitation buffer. Loading buffer was added to each sample and incubated at $95{ }^{\circ} \mathrm{C}$ for 15 minutes, centrifuged and subjected to SDS-PAGE. After appropriate separation, gels were transferred and analyzed for coimmunoprecipitation using standard immunoblotting techniques as detailed above.

\section{Statistical analysis}

All experiments were performed at least three times, and the mean and standard deviation (SD) are shown for each experiment. Statistical analysis was carried out in Excel, Prism GraphPad 5 and SPSS. Statistical analysis was done via Student $t$-test, chi-square test, ANOVA, and CochranArmitage test for trend. Significance was defined as $\mathrm{P}<0.05$.

\section{Results}

\section{Rab25 predicts poor prognosis in $G A C$}

To observe the expression level of Rab25 in GAC tissues, immunohistochemical staining was used in the various differentiated GAC tissues. The Rab25 immunohistochemistry (IHC) score was significantly higher in GAC tissue than in paracancerous tissue (Figure 1A). In addition, the staining degree of Rab25 protein in GAC tissue was negatively correlated with the degree of differentiation. The highly differentiated GAC tissues were weakly stained while the poorly differentiated GAC tissues were strongly stained. It appeared that the poorer the level of differentiation, the stronger the degree of immunohistochemical staining (Figure 1B). According to the IHC score of Rab25, we divided the patients into high expression group and low expression group. KaplanMeier 5-year survival analysis revealed a significantly reduced survival duration in GAC patients with high tumor Rab25 expression (Figure 1C). We further performed clinicopathologic correlation with Rab25 expression in GAC patients (Table 2). Our analysis confirmed that high Rab25 expression is associated with poor tumor cell differentiation.

\section{Silencing Rab25 inbibits GAC cells proliferation in vitro}

To estimate the expression levels of Rab25 in GAC cell lines, the gene level was evaluated by real-time PCR in the cancer cells. We found that the expression of Rab25 was generally increased in GAC cells. In particular, the expression level of Rab25 in the GAC cell AGS was 18.77 times higher than that in the normal gastric mucosal epithelial cell GES-1 (Figure 2A). In our previous study, we identified the promoter of the human Rab25 gene and illuminated the mechanism by which PKA regulates the expression of Rab25 in AGS cells (31). Therefore, AGS cells were used to explore the important role of Rab25 in GAC. To investigate the effect of Rab25 on the function of GAC cells, Rab25 expression in AGS cells was silenced by lentivirus-mediated transfection. Both two shRNA oligos could effectively decreased Rab25 expression at transcriptional and protein levels in AGS cells (Figure 2B). In order to simplify the experiment, we selected shRab25-1 for the next experiment. To investigate the effect of rab25 on cell proliferation capacity, cell count assay and colonyformation assay were performed and the results showed that inhibition of Rab25 expression attenuated cell proliferation obviously (Figure $2 C, D$ ). In addition, knocking down Rab25 also significantly reduced the colony formation ability of AGS cells in soft agar (Figure 2E). These data show that silencing Rab25 inhibits the proliferation of GAC cells and reduces the ability of tumorigenesis.

\section{Rab25 up-regulation in GES-1 cells promotes cell proliferation and migration}

To further explore the significance of Rab25 in gastric cells, we selected normal human gastric mucosal epithelial cell line GES-1 as control and constructed the stable Rab25overexpressing GES-1 cells (Figure $3 A$ ). As shown in Figure $3 B$, cell count assay revealed that up-regulation of Rab25 expression significantly enhanced cell proliferation 
A

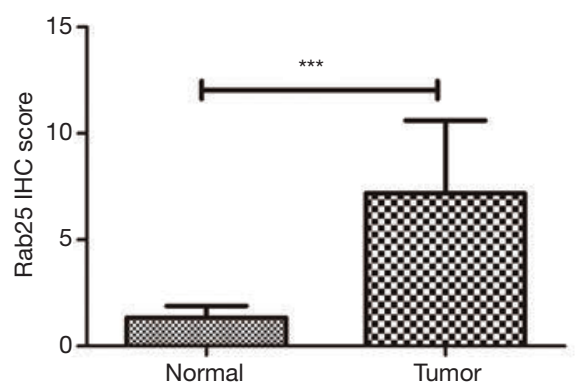

C

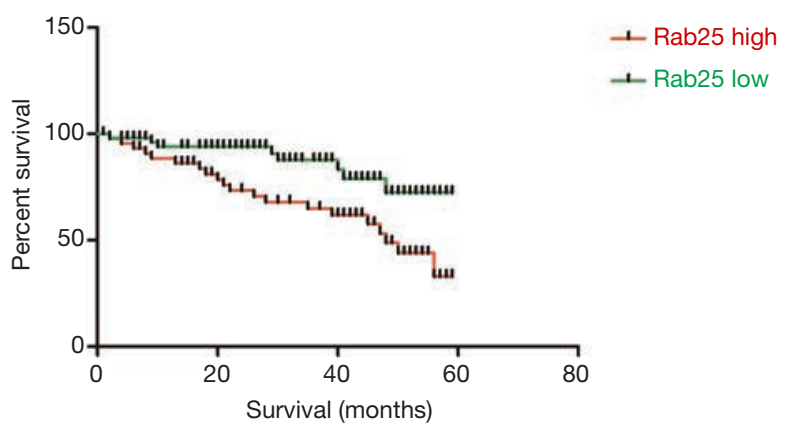

B
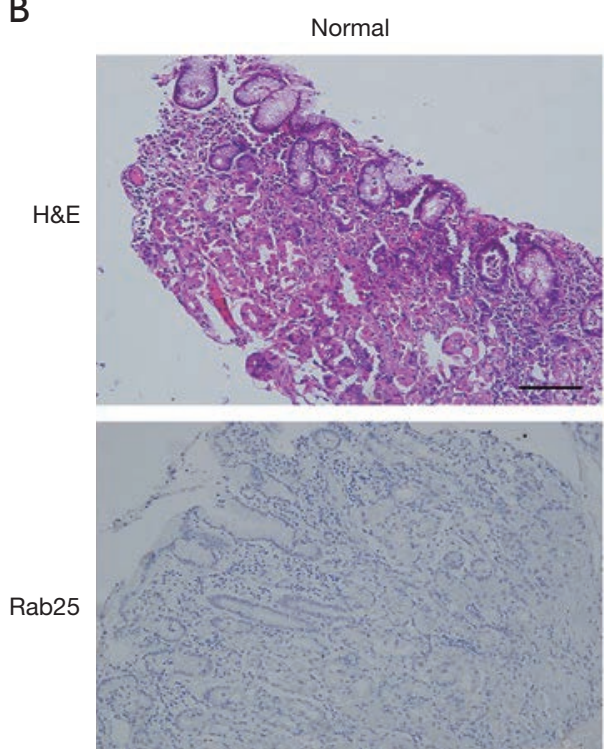
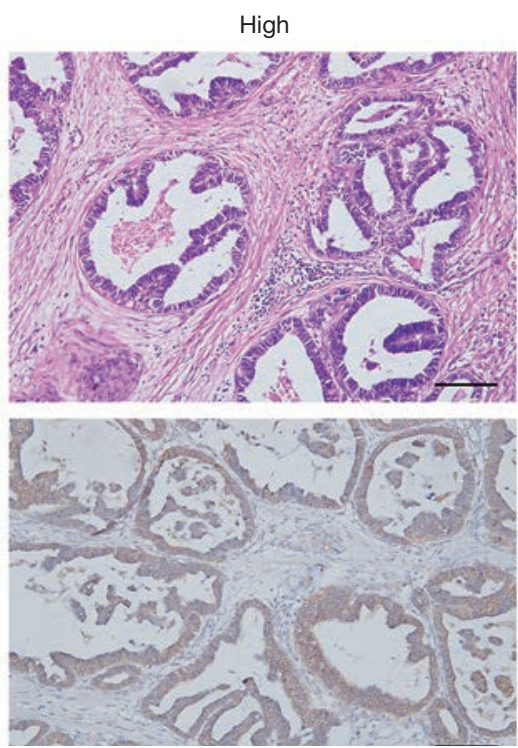

Poor
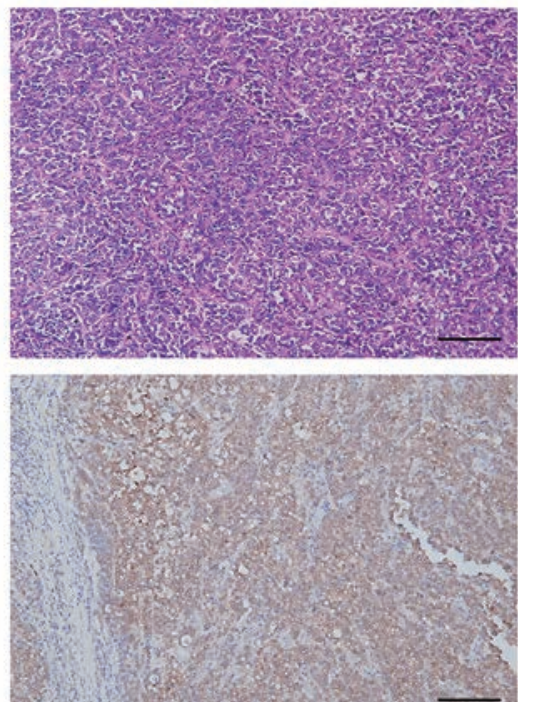

Figure 1 Rab25 predicts poor prognosis in gastric adenocarcinoma. (A) Comparison of IHC quantification of Rab25 expression from human gastric adenocarcinoma samples and matched normal gastric tissue samples ( $n=154)$. ${ }^{* * *}, \mathrm{P}<0.001$. (B) IHC staining of Rab25 proteins in human normal gastric tissues versus in human gastric adenocarcinoma samples. (C) Kaplan-Meier survival curves of 154 patients with gastric adenocarcinoma whose tumors had a high or low Rab25 expression. Rab25 high and low groups were separated by the median expression. Significance was determined with log-rank test. $\mathrm{P}=0.009$. Scale bars, $200 \mu \mathrm{m}$.

in pHBLV-Rab25 GES-1 cells compared with the parental pHBLV-n.c GES-1 cells. Furthermore, colony-formation assay also demonstrated that overexpression of Rab25 promotes cell proliferation in GES-1 cells (Figure 3C). To further examine the functional role of Rab25 in the GES1 cells, we performed the wound healing assay to evaluate the ability of cell migration. Microscopic examinations at $0,6,12$ hours revealed obvious difference of migration of GES-1 cells in the pHBLV-Rab25 group compared with controls (Figure 3D). At the same time, we found that overexpression of Rab25 promotes the colony formation ability of GES-1 cells in soft agar (Figure 3E). Collectively, these results suggest that Rab25 promotes cell proliferation and migration.

\section{Rab25 participates in the regulation of aerobic glycolysis in GAC cells}

High lactate production is characteristic of cancer cells exhibiting the Warburg effect. Accordingly, we tested the metabolic state of Rab25-knockdown AGS cells and pHBLV-Rab25 GES-1 cells by measuring their lactate levels using a Lactate Assay Kit. In the meantime, we detected the expression of pyruvate, the key metabolites involved in aerobic glycolysis. In Rab25-knockdown AGS cells, the level of pyruvate dropped by 4 times and the level of lactic acid 
Table 2 Clinical characteristics of patients based on different Rab25 expression

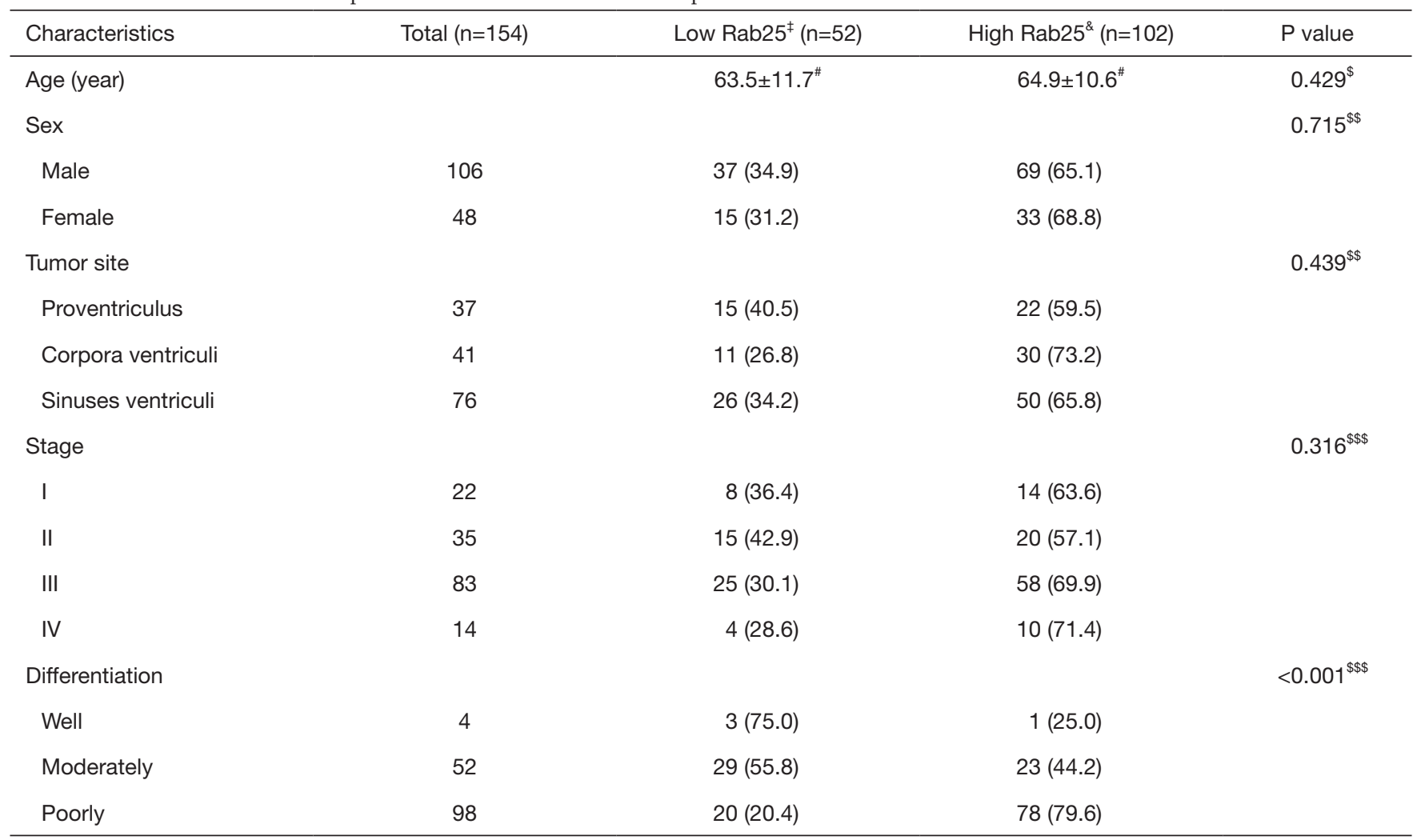

${ }^{\ddagger}$, IHC score: $0-5 ;{ }^{~}{ }^{2}$ IHC score: $6-12 ;{ }^{\#}$, mean \pm SD); ${ }^{\$}, t$-test; ${ }^{\$ \$}$, chi-square test; ${ }^{\$ \$}$, Cochran-Armitage test for trend.

decreased by approximately 1.6 times, when compared with those in the controls respectively (Figure 4A), suggesting that Rab25 is a positive regulator of glycolysis. Meanwhile, in pHBLV-Rab25 GES-1 cells, the level of pyruvate and lactic acid increased with the overexpression of Rab25 when compared with those in the controls respectively (Figure 4B). Taken together, these results validated that Rab25 participated in the regulation of aerobic glycolysis in GAC cells. On this basis, we envisioned a model diagram of Rab25 participating in the regulation of aerobic glycolysis and made a preliminary exploration (Figure $4 C$ ).

\section{Rab25 is a positive regulator of PKM2}

To find out the specific mechanism of Rab25 participating in the regulation of Warburg effect, we analyzed the relationship between Rab25 and the key molecules in aerobic glycolysis pathway by utilizing GRAMM-X proteinprotein docking database (32). The result reflects there may be an interaction between Rab25 and PKM2. The PKM2 IHC score was significantly higher in GAC tissue than in paracancerous tissue (Figure $5 A, B$ ). To further search the effect of Rab25 on the expression of PKM2, analysis of IHC scores in 41 patients was performed to examine the correlation between Rab25 with PKM2. Our results demonstrated that PKM2 expression is positively correlated with the expressions of Rab25 and the correlation coefficient (R) was 0.818 (Figure 5C). Then the immunofluorescent staining showed that Rab25 protein and PKM2 protein co-located on the cell membrane in AGS cells (Figure 5D). At the same time, we found that Rab25 protein and PKM2 protein could bind to each other by coimmunoprecipitation in AGS cells (Figure 5E). To further confirm the effect of Rab25 on the expression of PKM2, we detected the activity of PK and the gene expression level of PKM2 in the stable Rab25-overexpressing and Rab25silencing cells (Figure 5F,G). Through detection, we found that the activity of PK and the gene level of PKM2 were positively related to Rab25. The results reflect that Rab25 has a positive regulatory effect on PKM2. Moreover, we transfect AGS stable transfected cells with Control siRNA (siCtrl) and PKM2 siRNA (siPKM2) and use plate cloning 
A

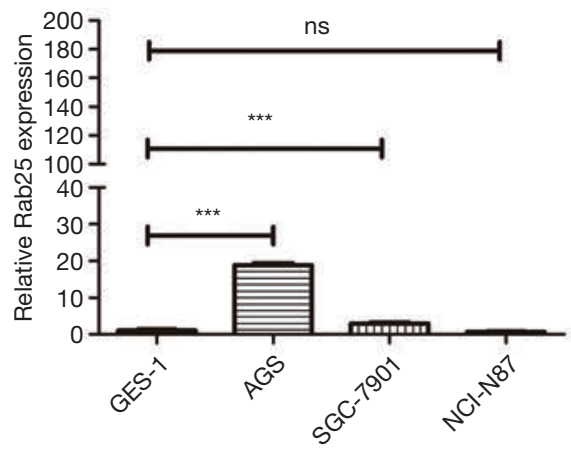

B

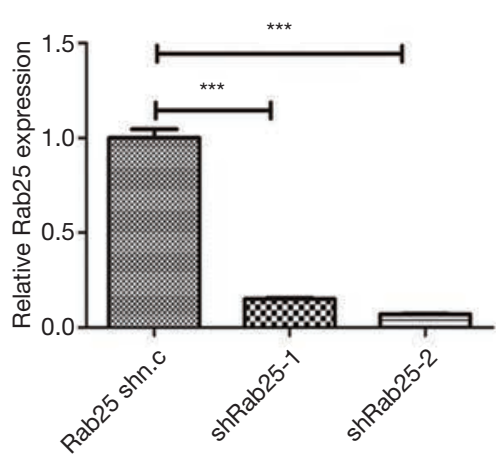

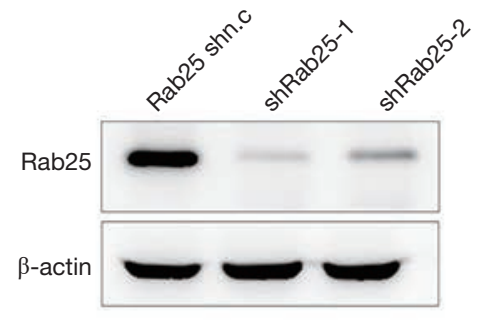

$c$

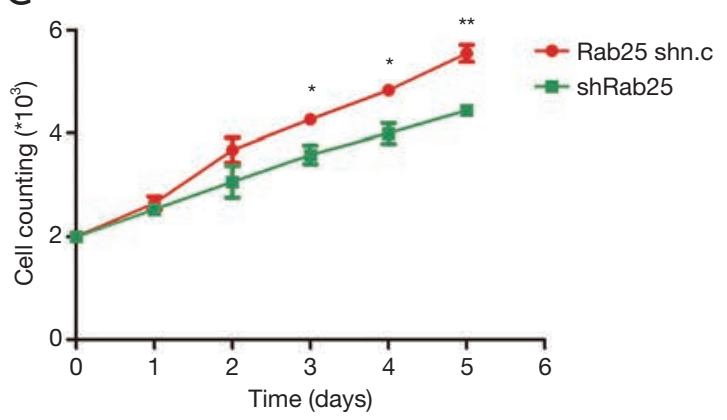

$\mathrm{D}$

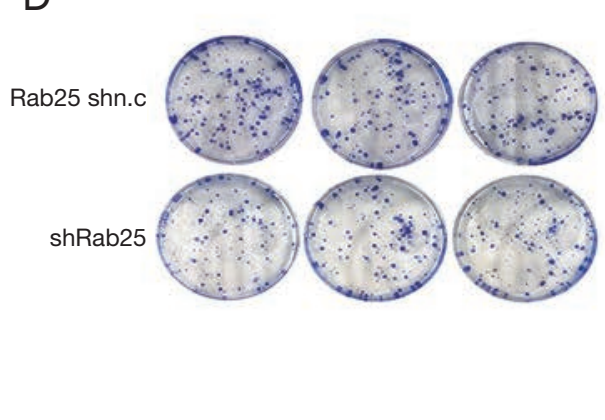

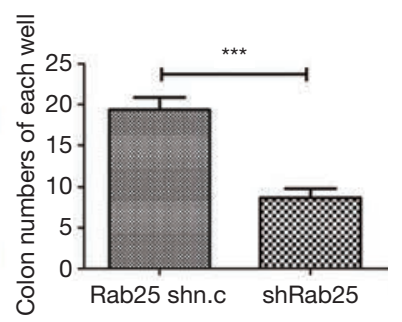

E
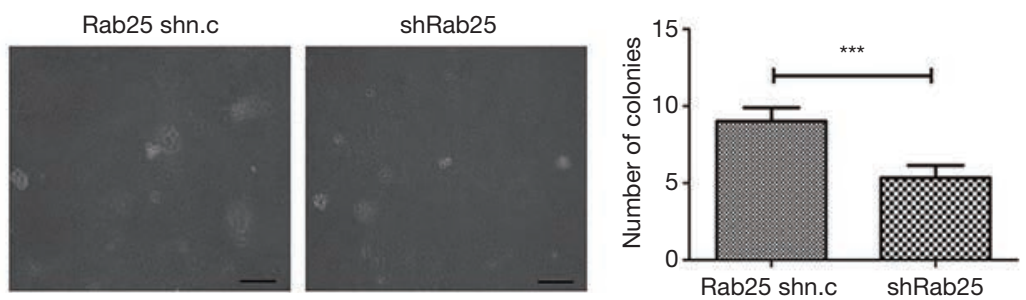

Figure 2 Silencing Rab25 inhibits gastric adenocarcinoma cells proliferation in vitro. (A) qPCR analysis comparing Rab25 mRNA expression (normalized to $\beta$-actin) in gastric adenocarcinoma cells with normal gastric mucosal epithelial cell lines GES-1. ***, $\mathrm{P}<0.001$; ns, not statistically significant. (B) qPCR analysis (normalized to $\beta$-actin) and western blot analysis (normalized to $\beta$-Actin) of Rab25 expression in AGS cells transfected with control shRNA (Rab25 shn.c) or two different shRNAs against Rab25 (shRab25-1 and shRab25-2). ***, $\mathrm{P}<0.001$. (C) The growth curves of the Rab25 shn.c and shRab25 AGS cells by survival cell count assay in vitro. * $\mathrm{P}<0.05 ;{ }^{* *}, \mathrm{P}<0.01$. (D) Representative images (left) and quantification (right) of colony formation in six-well plates from the Rab25 shn.c and shRab25 AGS cells. ***, $\mathrm{P}<0.001$. Stained by coomassie brilliant blue dye and clones in 6-well plate without magnificatio. (E) Representative images (left) and quantification (right) of colony formation per 10-fold field of view in soft agar from the Rab25 shRNA knockdown AGS cell lines. ***, $\mathrm{P}<0.001$. Stained and magnified ten times.

experiments to detect changes in cell proliferation capacity. The results show that the activity of PKM2 is necessary for the oncogenic functions of Rab25 (Figure $5 \mathrm{H}$ ).

\section{Rab25 up-regulation promotes phosphorylation of PKM2}

Given that phosphorylation of PKM2 protein plays a key role in the regulation of PKM2 activity (33), we focused on the effect of Rab25 on PKM2 phosphorylation. Indeed, we found that in vitro, western blotting showed that Rab25 could significantly promote the phosphorylation level of tyrosine of PKM2 protein (PKM2-pTyr) (Figure 6A,B). It is known that multiple kinases frequently activated in different cancer types were identified to phosphorylate 
A
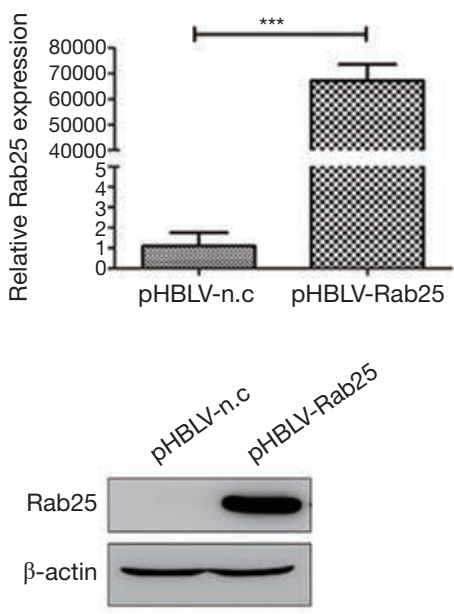

B
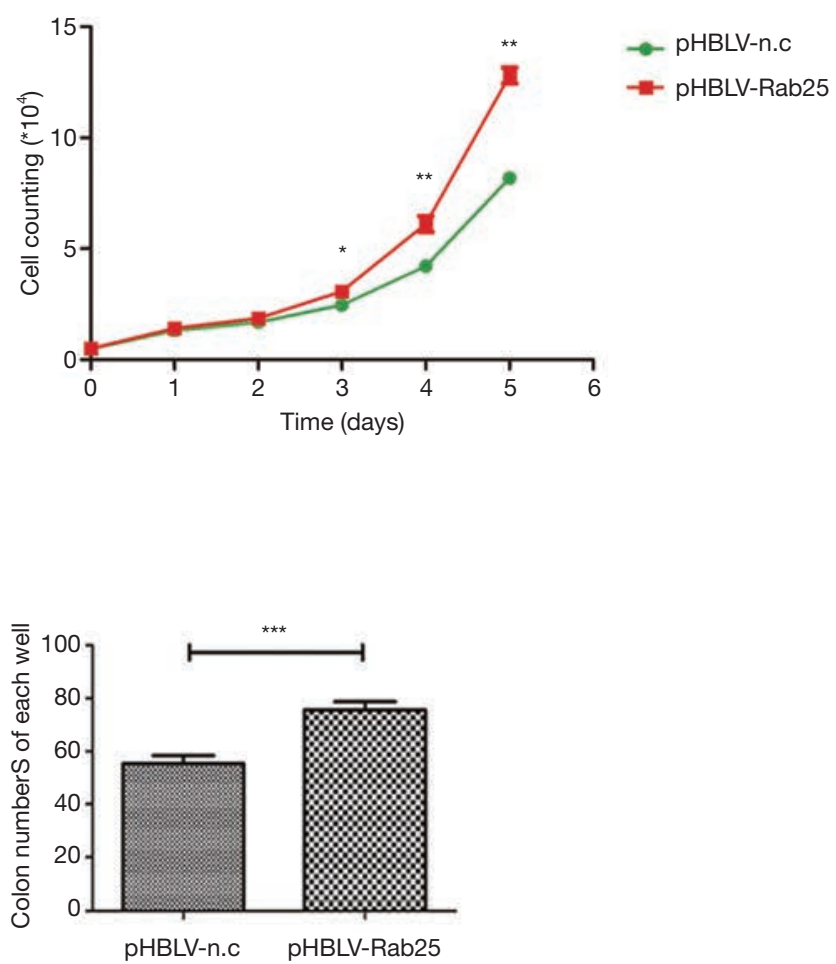

$\mathrm{D}$

C
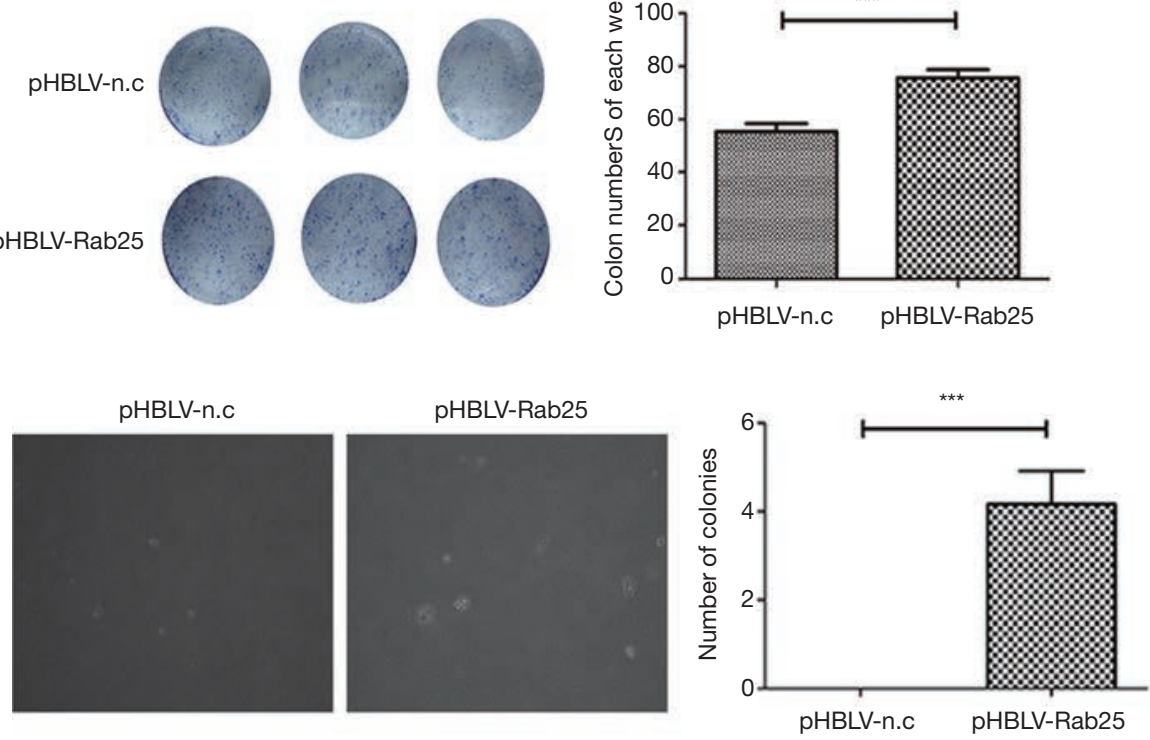

E

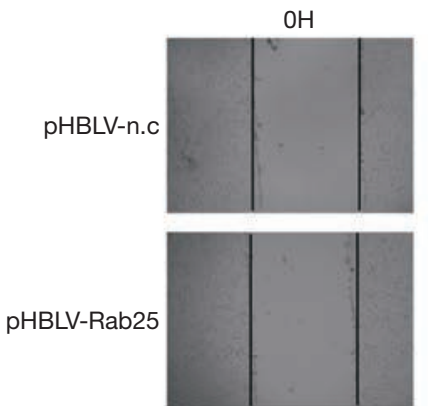

$6 \mathrm{H}$
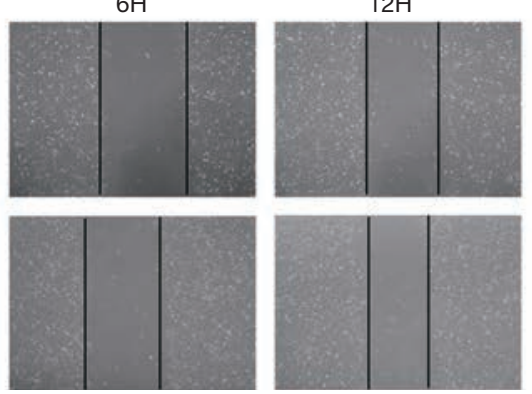

Figure 3 Rab25 up-regulation in GES-1 cells promotes cell proliferation and migration. (A) qPCR analysis (normalized to $\beta$-actin) and western blot analysis (normalized to $\beta$-actin) of Rab25 expression in GES-1 cells transfected with pHBLV-n.c or pHBLV- Rab25 lentivirus. ***, $\mathrm{P}<0.001$. (B) The growth curves of the pHBLV-n.c and pHBLV-Rab25 GES-1 cells by survival cell count assay in vitro. *, $\mathrm{P}<0.05$; **, $\mathrm{P}<0.01$. (C) Representative images (left) and quantification (right) of colony formation in six-well plates from the Rab25 overexpression GES-1 sublines. ***, $\mathrm{P}<0.001$. Stained by coomassie brilliant blue dye and clones in 6-well plate without magnification. (D) Representative images (left) and quantification (right) of colony formation per 10-fold field of view in soft agar from the pHBLV-n.c and pHBLV-Rab25 GES-1 cells. ***, $\mathrm{P}<0.001$. (E) Cell migration was detected using wound healing assay to investigate the effect of Rab25 in GES-1 cells. Photographs were taken at 0,6 , and 12 hours. Stained and magnified ten times. 


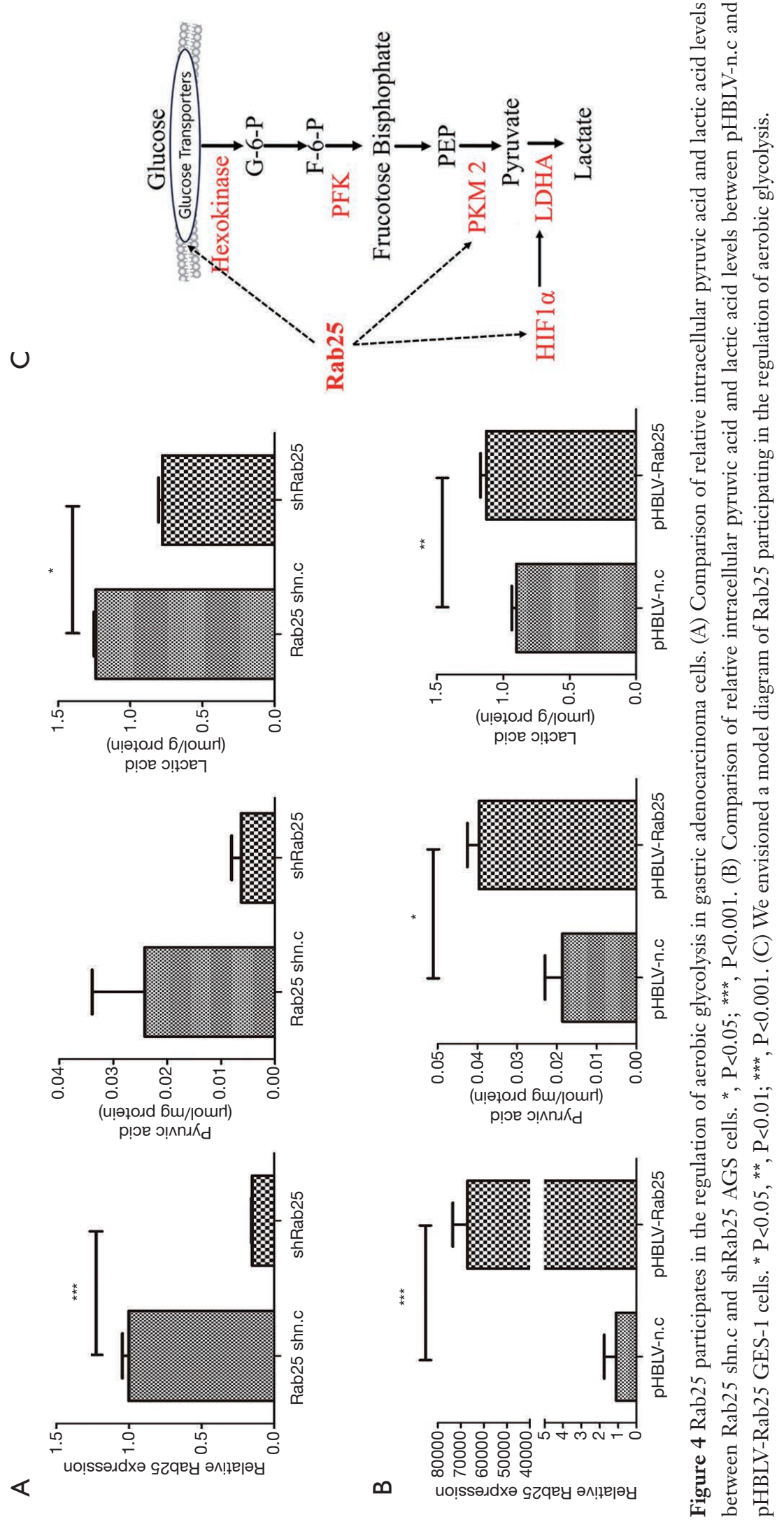



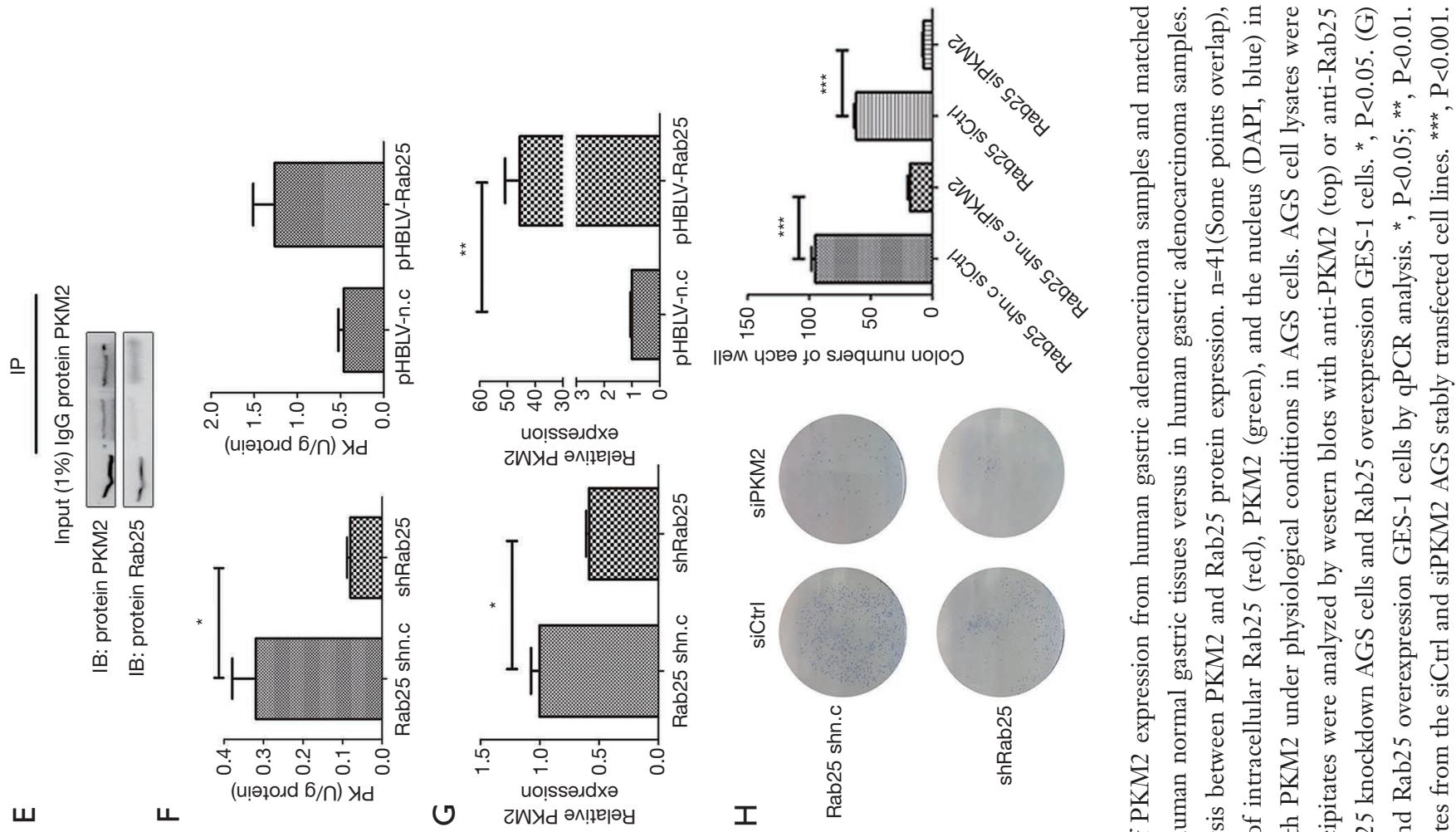

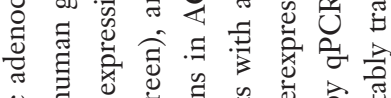

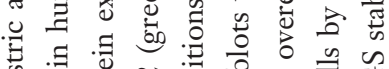

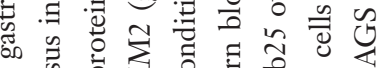

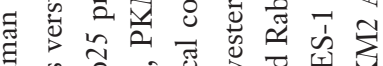

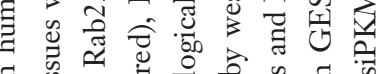

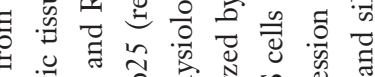

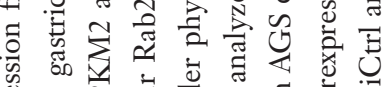

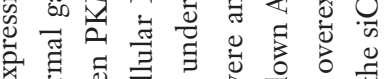

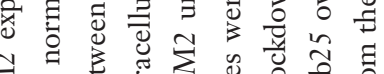

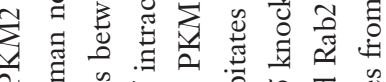
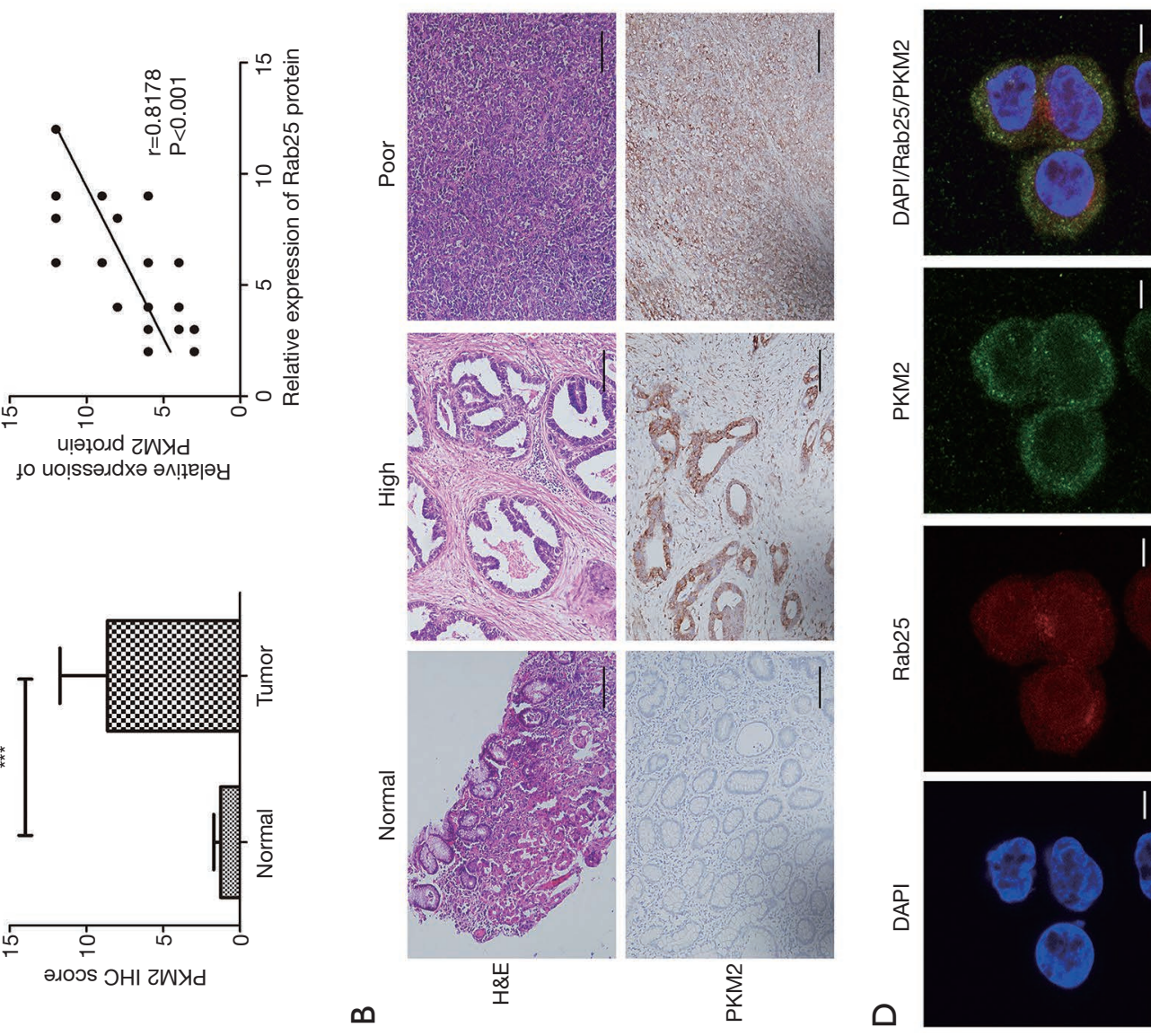

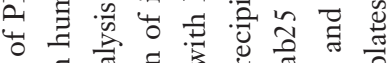

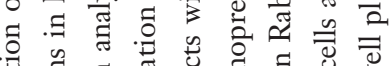

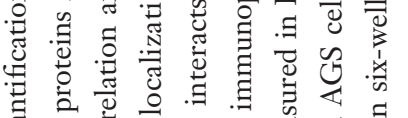
ปี

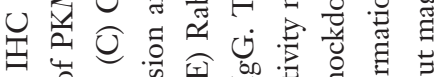

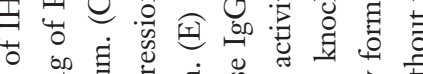

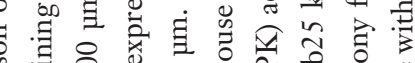

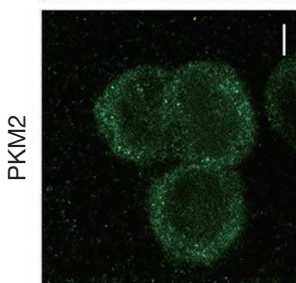

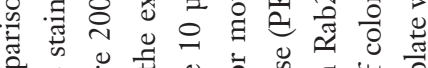

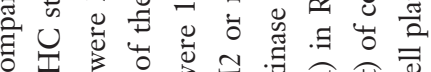

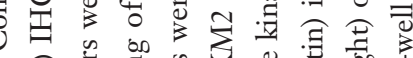

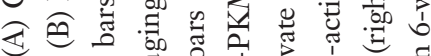
¿் U

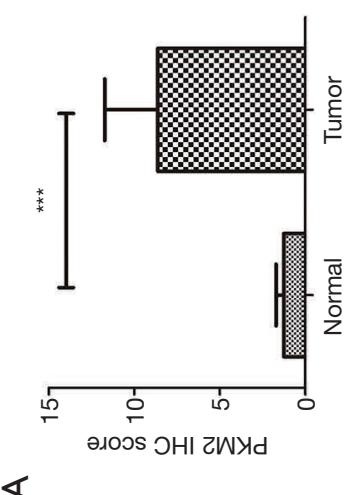

$\infty$

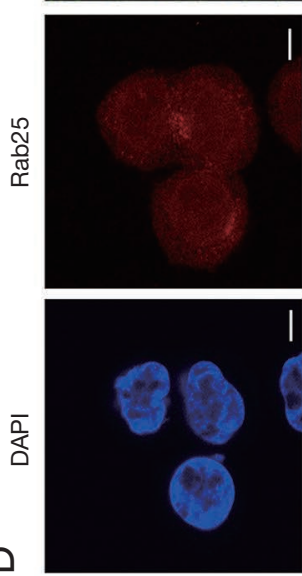

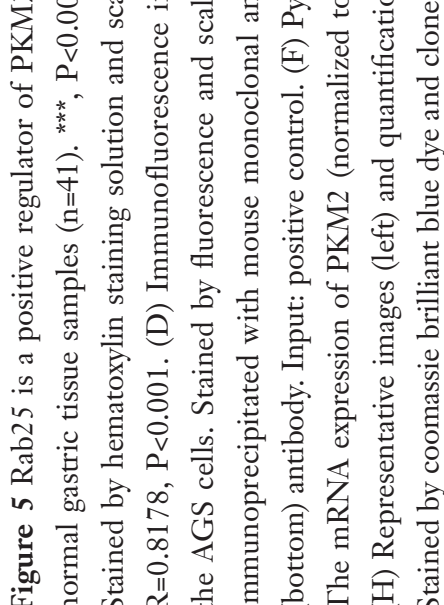


A

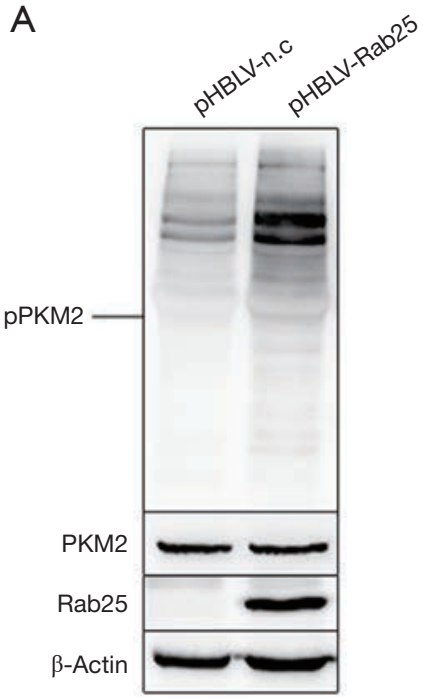

B

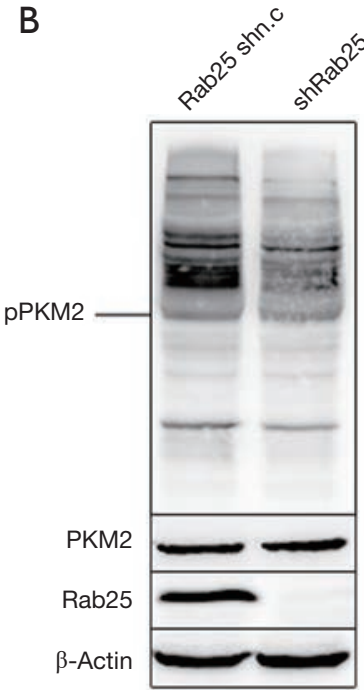

C
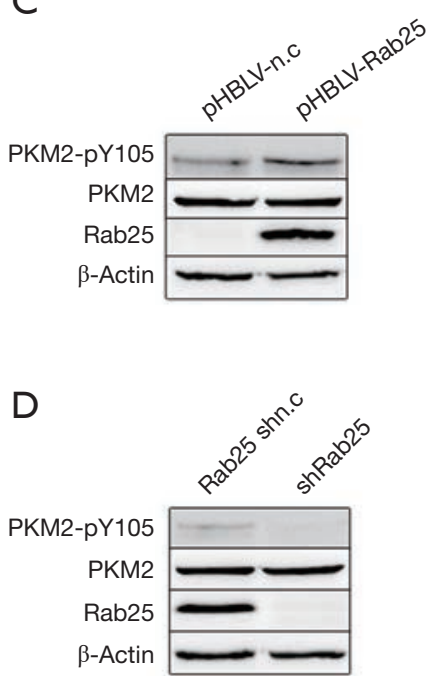

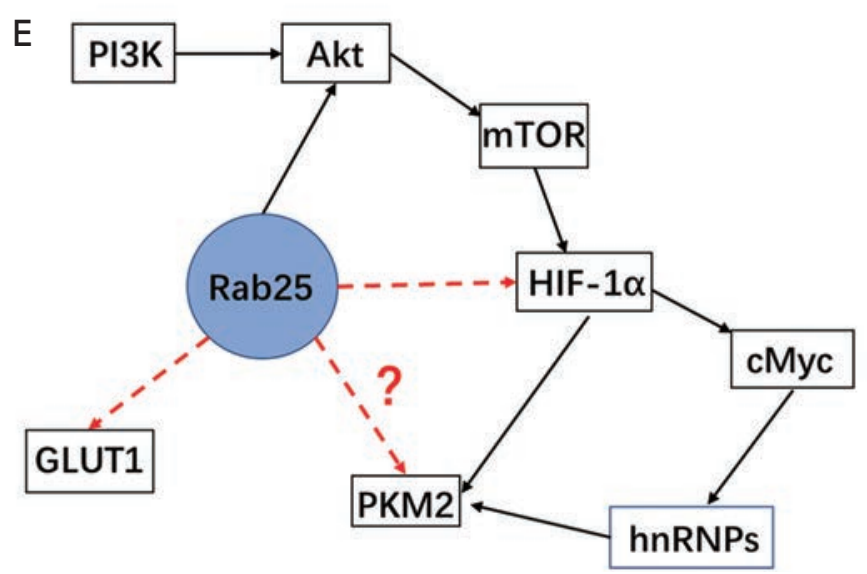

Figure 6 Rab25 up-regulation promotes phosphorylation of PKM2. (A) Western blot analysis (normalized to $\beta$-Actin) of PKM2-pTyr, PKM2, and Rab25 protein levels in the pHBLV-n.c and pHBLV-Rab25 GES-1 cells. (B) Western blot analysis (normalized to $\beta$-Actin) of PKM2-pTyr, PKM2, and Rab25 protein levels in the Rab25 shn.c and shRab25 AGS cells. (C) Western blot analysis (normalized to $\beta$-Actin) of PKM2-pY105, PKM2, and Rab25 protein levels in the pHBLV-n.c and pHBLV-Rab25 GES-1 cells. (D) Western blot analysis (normalized to $\beta$-Actin) of PKM2- pY105, PKM2, and Rab25 protein levels in the Rab25 shn.c and shRab25 AGS cells. (E) An assumed diagram of the mechanism of Rab25 regulating glycolysis pathway in gastric adenocarcinoma cells.

PKM2-Y105 (34). Therefore, we focused on the Y105 site and found that Rab25 could significantly promote the phosphorylation level of PKM2-Y105 in vitro (PKM2pY105) (Figure 6C,D). Taken together, these data suggest that Rab25 is involved in regulating the Warburg effect by phosphorylating PKM2-Y105.

\section{Discussion}

In this study, we have discovered that Rab25 was highly expressed in GAC tissues and cells. Meanwhile, 5-year survival analysis revealed a significantly reduced survival duration in GAC patients with high tumor Rab25 expression. Our results demonstrated that silencing Rab25 expression attenuated cell proliferation and clone formation capacity in GAC cells (AGS) in vitro. To explore the underlying mechanism, we analyzed the regulatory function of Rab25 in aerobic glucose metabolism, a process that plays an important role in the development and transformation of malignant tumors (35-37). As demonstrated, silencing 
Rab25 inhibited the Warburg effect in GAC cells. Moreover, the expressions of relevant glycolytic genes including PKM2, GLUT1, HK2, and HIF- $1 \alpha$ were decreased in Rab25-knockdown GAC cells (Figure S1). Rab25 may form complexes with other proteins, and bind to other receptors in synergy with endogenous and exogenous danger signals to regulate Warburg effect. As is widely acknowledged, PI3K/AKT/mTOR pathway is one of the important signal transduction pathways in cells (38). Many studies have shown that mTOR/HIF-1 $\alpha /$ Myc-hnRNPs/ PKM2 signaling pathway plays an extremely important role in glycolysis pathway $(39,40)$. Meanwhile, Rab25 can combines with AKT (41) and we drew a diagram of the mechanism of Rab25 regulating glycolysis pathway in GAC cells (Figure 6E). In this study, our results demonstrated that Rab25 has a positive regulatory effect on PKM2. Since phosphorylation of PKM2 protein plays a key role in the regulation of PKM2 activity, it is reasonable to speculate that Rab25 may have an effect on PKM2 phosphorylation. Phosphorylation experiments showed that Rab25 could significantly promote the level of tyrosine phosphorylation of PKM2 protein in vitro. In addition, phosphorylation of PKM2-Y105 was shown to correlate with tumor progression (33) and PKM2-pY105 was also shown to play a key role in cell transformation (34), we focused on the Y105 site and found that Rab25 could significantly promote the phosphorylation level of PKM2-Y105 in vitro. Taken together, we propose that Rab25 is involved in regulating the Warburg effect via phosphorylating PKM2-Y105 in GAC.

Many studies have shown that Rab25 acts as an oncogene in most epithelial tumors including ovarian $(13,14)$, luminal B breast (15), testicular (16), bladder (17), prostatic (18) and liver tumors (19). Our findings have revealed that Rab25 acts as an oncogene in GAC, which also belongs to an epithelial tumor. In addition, our previous study has elucidated the mechanism of increased gene expression of Rab25 in GAC cells (AGS) (31). Rab25 was reported to promote tumor growth or metastasis in ovarian (42) and bladder cancer (17) at increased expression level. We selected normal human gastric mucosal epithelial cell line GES-1 as control and constructed the stable Rab25-overexpressing GES-1 cells. With Rab25 expression increased in normal human gastric mucosal epithelial cells (GES-1), the ability of proliferation and migration of gastric cells have improved significantly. Furthermore, overexpression of Rab25 may induce normal gastric cells to have a tendency for malignancy. On the one hand, in this study we found a significant increase in the level of HIF-1 $\alpha$ gene in gastric cells overexpressing Rab25 (Figure S2) and the biological effects mediated by HIF$1 \alpha$ are thought to be conducive to cancer development and progression (43). On the other hand, we found that overexpression of Rab25 promotes the colony formation ability of GES-1 cells in soft agar. Furthermore, recent research has shown that PKM2 has dichotomous functions in nontransformed cells (nononcogenic) versus in cancer cells (tumorigenic). In nontransformed mammary epithelial cells, unphosphorylated PKM2 proteins form tetramers with high PK activity. However, in transformed tumor cells, PKM2 proteins are phosphorylated at Y105 and dissociate into dimers. The pY105-PKM2 promotes YAP nuclear translocation and increases cancer stem-like cells, becoming essential for tumor cell growth and transformation (34). These results can support that PKM2 proteins are phosphorylated at Y105 by Rab25 and induce GES-1 cells (nononcogenic) have a tendency for malignancy found in our research.

As demonstrated, Rab25 is involved in the regulation of aerobic glycolysis and plays a positive role. PKM2, GLUT1 and HIF- $1 \alpha$ are the key molecules in glycolysis pathway and we had conceived that there are three regulatory pathways for Rab25 to participate in aerobic glycolysis (Figures $4 C, 6 E$ ). But in our study, there was no significant correlation between the expression of Rab25 and GLUT1 (Figures S1,S2). At the same time, there was a positive correlation between the expression of HIF- $1 \alpha$ and Rab25 (Figures S1,S2). Further screening for Rab25 interaction partners using mass spectrometry is required to further explore the function of Rab25 in GAC.

The intervention of tumor metabolic dysregulations has been considered as a promising strategy for cancer therapy. However, direct modulation of PKM2 may destruct the balance of metabolism (44). However, there are more and more data indicating that oncogenes and tumor-related signaling pathways are more specific to the regulation of enzymes in glucose metabolism (29). Therefore, targeting oncogenic activation of PKM2 could be an alternative effective strategy. Recent studies have shown that aberrant cancer metabolism is closely related to chemotherapeutic resistance (45). In this study, we demonstrated that Rab25 is a positive regulator of aerobic glycolysis in GAC. Based on the contribution of aberrant metabolism to chemotherapeutic resistance, it is possible that silencing Rab25 functions may contribute to the improvement of treatment. Further studies are required to use Rab25 as an intervention target for improving GAC treatment. 
In summary, we demonstrated that Rab25 acts as an oncogene and predicts poor prognosis in GAC. In this study, we elucidated the specific mechanism that Rab25 participates in the regulation of aerobic glycolysis via PKM2 in GAC. The observation will expand the understanding of the function of Rab25 and the understanding of glycolytic regulatory network containing PKM2 protein in tumor cells. Our results may provide brand new perspectives on elucidating gastric carcinogenesis in terms of the effects of tumor genes on Warburg effects and thus more efficacious molecular targets for the treatment of gastric cancer.

\section{Acknowledgments}

Funding: This work was supported by the National Natural Science Foundation of China (Grant No. 81830052, 81530053)and the Natural Science Foundation of Shanghai (Grant No. 14ZR1425100).

\section{Footnote}

Reporting Checklist: The authors have completed the MDAR checklist. Available at http://dx.doi.org/10.21037/tcr-202597

Data Sharing Statement: Available at http://dx.doi. org/10.21037/tcr-20-2597

Conflicts of Interest: All authors have completed the ICMJE uniform disclosure form (available at http://dx.doi. org/10.21037/tcr-20-2597). The authors have no conflicts of interest to declare.

Ethical Statement: The authors are accountable for all aspects of the work in ensuring that questions related to the accuracy or integrity of any part of the work are appropriately investigated and resolved.

Open Access Statement: This is an Open Access article distributed in accordance with the Creative Commons Attribution-NonCommercial-NoDerivs 4.0 International License (CC BY-NC-ND 4.0), which permits the noncommercial replication and distribution of the article with the strict proviso that no changes or edits are made and the original work is properly cited (including links to both the formal publication through the relevant DOI and the license). See: https://creativecommons.org/licenses/by-nc-nd/4.0/.

\section{References}

1. Bray F, Ferlay J, Soerjomataram I, et al. Global cancer statistics 2018: GLOBOCAN estimates of incidence and mortality worldwide for 36 cancers in 185 countries. CA Cancer J Clin 2018;68:394-424.

2. Ajani JA, Lee J, Sano T, et al. Gastric adenocarcinoma. Nat Rev Dis Primers 2017;3:17036.

3. Ward PS, Thompson CB. Metabolic reprogramming: a cancer hallmark even warburg did not anticipate. Cancer Cell 2012;21:297-308.

4. Hanahan D, Weinberg RA. Hallmarks of cancer: the next generation. Cell 2011;144:646-74.

5. Warburg O. On the origin of cancer cells. Science 1956;123:309-14.

6. Novick P, Zerial M. The diversity of Rab proteins in vesicle transport. Curr Opin Cell Biol 1997;9:496-504.

7. Goldenring JR, Shen KR, Vaughan HD, et al. Identification of a small GTP-binding protein, Rab25, expressed in the gastrointestinal mucosa, kidney, and lung. J Biol Chem 1993;268:18419-22.

8. Mitra S, Montgomery JE, Kolar MJ, et al. Stapled peptide inhibitors of RAB25 target context-specific phenotypes in cancer. Nat Commun 2017;8:660.

9. Cheng KW, Lahad JP, Kuo WL, et al. The RAB25 small GTPase determines aggressiveness of ovarian and breast cancers. Nat Med 2004;10:1251-6.

10. Hu C, Chen B, Zhou Y, et al. High expression of Rab25 contributes to malignant phenotypes and biochemical recurrence in patients with prostate cancer after radical prostatectomy. Cancer Cell Int 2017;17:45.

11. Goldenring JR, Nam KT. Rab25 as a tumour suppressor in colon carcinogenesis. Br J Cancer 2011;104:33-6.

12. Nam KT, Lee HJ, Smith JJ, et al. Loss of Rab25 promotes the development of intestinal neoplasia in mice and is associated with human colorectal adenocarcinomas. J Clin Invest 2010;120:840-9.

13. Cheng KW, Lu Y, Mills GB. Assay of Rab25 function in ovarian and breast cancers. Methods Enzymol 2005;403:202-15.

14. Liu Y, Tao X, Jia L, et al. Knockdown of RAB25 promotes autophagy and inhibits cell growth in ovarian cancer cells. Mol Med Rep 2012;6:1006-12.

15. Mitra S, Federico L, Zhao W, et al. Rab25 acts as an oncogene in luminal B breast cancer and is causally associated with Snail driven EMT. Oncotarget 2016;7:40252-65. 
16. Korkola JE, Heck S, Olshen AB, et al. In vivo differentiation and genomic evolution in adult male germ cell tumors. Genes Chromosomes Cancer 2008;47:43-55.

17. Zhang J, Wei J, Lu J, et al. Overexpression of Rab25 contributes to metastasis of bladder cancer through induction of epithelial-mesenchymal transition and activation of Akt/GSK-3 beta/Snail signaling. Carcinogenesis 2013;34:2401-8.

18. Calvo A, Xiao N, Kang J, et al. Alterations in gene expression profiles during prostate cancer progression: functional correlations to tumorigenicity and downregulation of selenoprotein-P in mouse and human tumors. Cancer Res 2002;62:5325-35.

19. Geng D, Zhao W, Feng Y, et al. Overexpression of Rab25 promotes hepatocellular carcinoma cell proliferation and invasion. Tumour Biol 2016;37:7713-8.

20. Schaner ME, Ross DT, Ciaravino G, et al. Gene expression patterns in ovarian carcinomas. Mol Biol Cell 2003;14:4376-86.

21. Cheng KW, Lahad JP, Gray JW, et al. Emerging role of RAB GTPases in cancer and human disease. Cancer Res 2005;65:2516-9.

22. Cheng KW, Agarwal R, Mitra S, et al. Rab25 increases cellular ATP and glycogen stores protecting cancer cells from bioenergetic stress. EMBO Mol Med 2012;4:125-41.

23. Israelsen WJ, Vander Heiden MG. Pyruvate kinase: Function, regulation and role in cancer. Semin Cell Dev Biol 2015;43:43-51.

24. Imamura K, Tanaka T. Pyruvate kinase isozymes from rat. Methods Enzymol 1982;90 Pt E:150-65.

25. Keller KE, Tan IS, Lee YS. SAICAR stimulates pyruvate kinase isoform M2 and promotes cancer cell survival in glucose-limited conditions. Science 2012;338:1069-72.

26. Goldberg MS, Sharp PA. Pyruvate kinase M2-specific siRNA induces apoptosis and tumor regression. J Exp Med 2012;209:217-24.

27. Christofk HR, Vander Heiden MG, Harris MH, et al. The M2 splice isoform of pyruvate kinase is important for cancer metabolism and tumour growth. Nature 2008;452:230-3.

28. Vander Heiden MG, Locasale JW, Swanson KD, et al. Evidence for an alternative glycolytic pathway in rapidly proliferating cells. Science 2010;329:1492-9.

29. Vander Heiden MG. Targeting cancer metabolism: a therapeutic window opens. Nat Rev Drug Discov 2011;10:671-84.

30. Goodwin J, Neugent ML, Lee SY, et al. The distinct metabolic phenotype of lung squamous cell carcinoma defines selective vulnerability to glycolytic inhibition. Nat Commun 2017;8:15503.

31. Xue H, Qiao Y, Ni P, et al. A CRE that binds CREB and contributes to PKA-dependent regulation of the proximal promoter of human RAB25 gene. Int J Biochem Cell Biol 2011;43:348-57.

32. Tovchigrechko A, Vakser IA. GRAMM-X public web server for protein-protein docking. Nucleic Acids Res 2006;34:W310-4.

33. Hitosugi T, Kang S, Vander Heiden MG, et al. Tyrosine phosphorylation inhibits PKM2 to promote the Warburg effect and tumor growth. Sci Signal 2009;2:ra73.

34. Zhou Z, Li M, Zhang L, et al. Oncogenic KinaseInduced PKM2 Tyrosine 105 Phosphorylation Converts Nononcogenic PKM2 to a Tumor Promoter and Induces Cancer Stem-like Cells. Cancer Res 2018;78:2248-61.

35. Végran F, Boidot R, Michiels C, et al. Lactate influx through the endothelial cell monocarboxylate transporter MCT1 supports an NF-kappaB/IL-8 pathway that drives tumor angiogenesis. Cancer Res 2011;71:2550-60.

36. Roy BC, Kohno T, Iwakawa R, et al. Involvement of LKB1 in epithelial-mesenchymal transition (EMT) of human lung cancer cells. Lung Cancer 2010;70:136-45.

37. Jeong BY, Cho KH, Jeong KJ, et al. Rab25 augments cancer cell invasiveness through a betal integrin/EGFR/ VEGF-A/Snail signaling axis and expression of fascin. Exp Mol Med 2018;50:e435.

38. Datta SR, Brunet A, Greenberg ME. Cellular survival: a play in three Akts. Genes Dev 1999;13:2905-27.

39. Luo $W, \mathrm{Hu} \mathrm{H}$, Chang $\mathrm{R}$, et al. Pyruvate kinase $\mathrm{M} 2$ is a PHD3-stimulated coactivator for hypoxia-inducible factor 1. Cell 2011;145:732-44.

40. Sun Q, Chen X, Ma J, et al. Mammalian target of rapamycin up-regulation of pyruvate kinase isoenzyme type M2 is critical for aerobic glycolysis and tumor growth. Proc Natl Acad Sci U S A 2011;108:4129-34.

41. Tang BL, Ng EL. Rabs and cancer cell motility. Cell Motil Cytoskeleton 2009;66:365-70.

42. Gomez-Roman N, Sahasrabudhe NM, McGregor F, et al. Hypoxia-inducible factor 1 alpha is required for the tumourigenic and aggressive phenotype associated with Rab25 expression in ovarian cancer. Oncotarget 2016;7:22650-64.

43. Jin F, Yang R, Wei Y, et al. HIF-1alpha-induced miR23 a approximately 27 a approximately 24 cluster promotes colorectal cancer progression via reprogramming 
metabolism. Cancer Lett 2019;440-441:211-22.

44. Anastasiou D, Yu Y, Israelsen WJ, et al. Pyruvate kinase M2 activators promote tetramer formation and suppress tumorigenesis. Nat Chem Biol 2012;8:839-47.

Cite this article as: Zheng T, Huang L, Chen N, Xue H, Ni P, Huang G. Rab25 acts as an oncogene and participates in the regulation of aerobic glycolysis via PKM2 in gastric adenocarcinoma. Transl Cancer Res 2021;10(2):790-805. doi: $10.21037 /$ tcr-20-2597
45. Xu J, Wang J, Xu B, et al. Colorectal cancer cells refractory to anti-VEGF treatment are vulnerable to glycolytic blockade due to persistent impairment of mitochondria. Mol Cancer Ther 2013;12:717-24. 


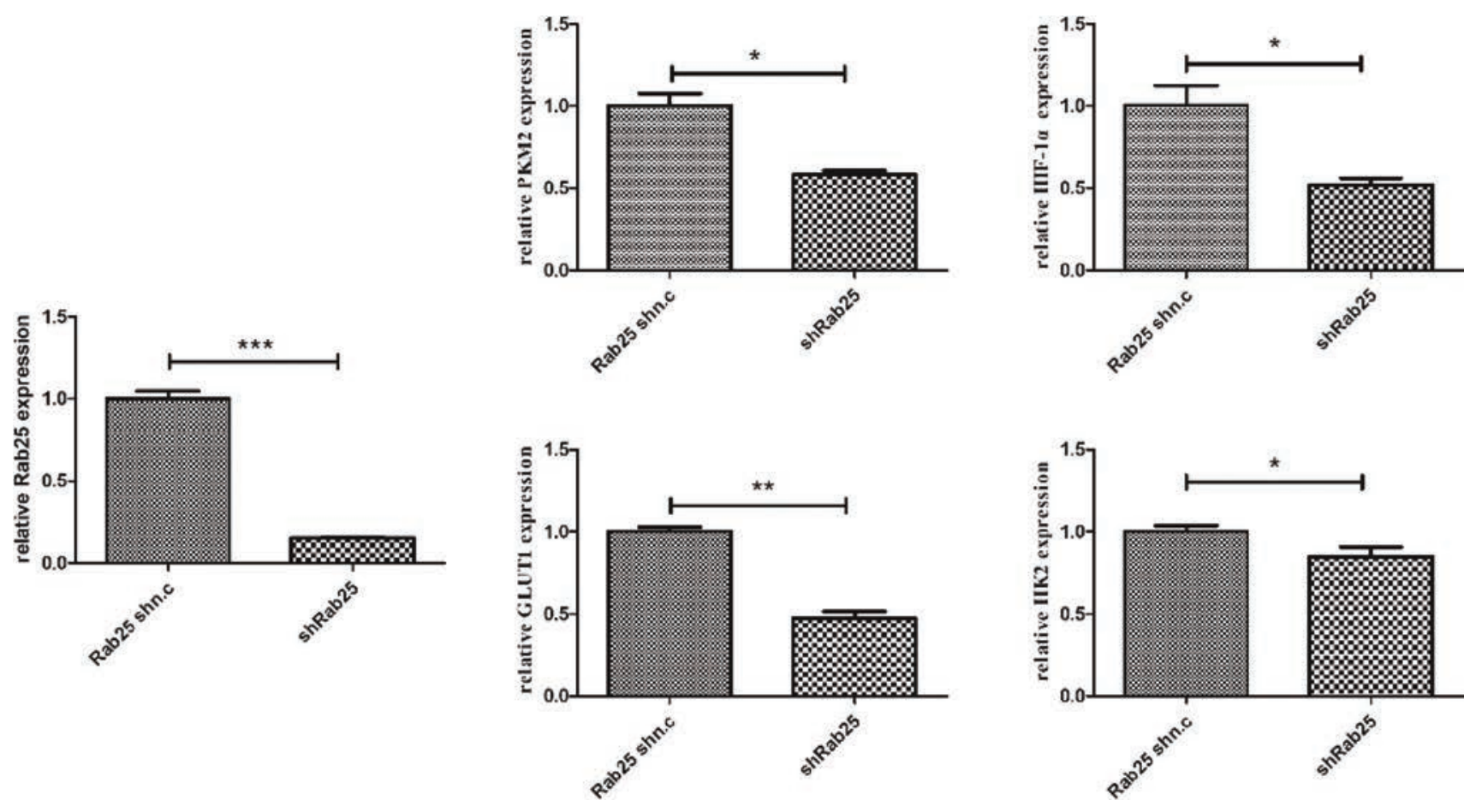

Figure S1 qPCR analysis comparing Rab25, PKM2, GLUT1 and HK2 mRNA expression (normalized to $\beta$-actin) in the Rab25 shn.c and shRab25 AGS cells. ${ }^{*} \mathrm{P}<0.05,{ }^{* *} \mathrm{P}<0.01,{ }^{* * *} \mathrm{P}<0.001$.
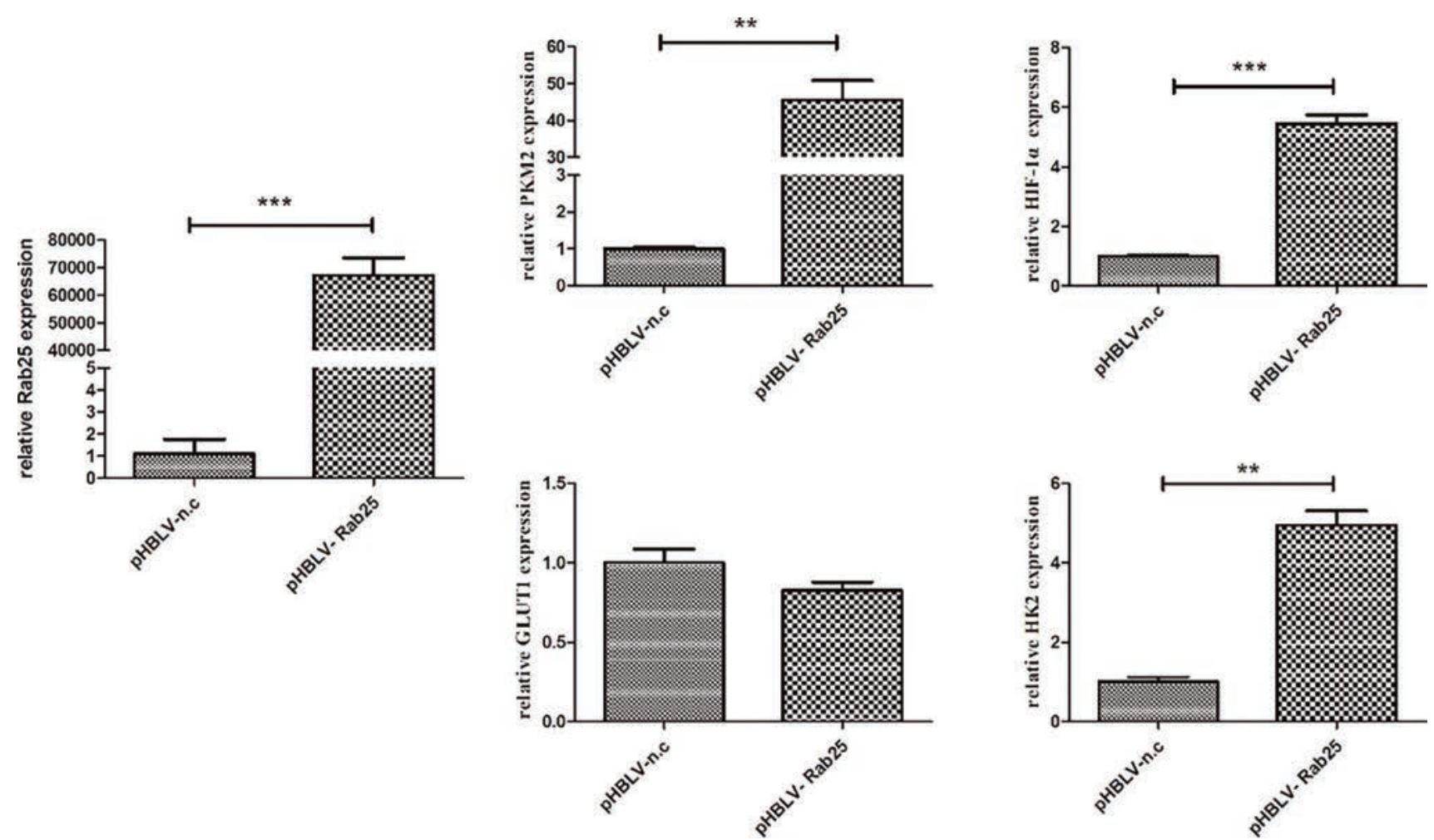

Figure S2 qPCR analysis comparing Rab25, PKM2, GLUT1 and HK2 mRNA expression (normalized to $\beta$-actin) in the pHBLV-n.c and pHBLV-Rab25 GES-1 cells. ${ }^{* *} \mathrm{P}<0.01,{ }^{* * *} \mathrm{P}<0.001$. 